\title{
Up-regulated osteogenic transcription factors during early response of human periodontal ligament stem cells to cyclic tensile strain
}

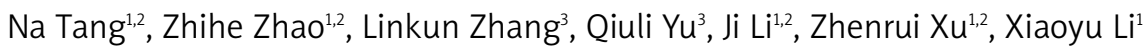

1State Key Laboratory of Oral Biomedical Engineering, Sichuan University, China 2Department of Orthodontics, West China College of Stomatology, Sichuan University, China

3Tianjin Stomatological Hospital, Nankai University, China

Submitted: 20 May 2011

Accepted: 4 September 2011

Arch Med Sci 2012; 8, 3: 422-430

DOI: 10.5114 /aoms.2012.28810

Copyright ( 2012 Termedia \& Banach

\section{Abstract}

Introduction: As one group of periodontal ligament (PDL) cells, human periodontal ligament stem cells (hPDLSCs) have been isolated and identified as mesenchymal adult stem cells (MSCs) since 2004. It has been well accepted that PDL sensitively mediates the transmission of stress stimuli to the alveolar bone for periodontal tissue remolding. Besides, the direction of MSCs differentiation has been verified regulated by mechanical signals. Therefore, we hypothesized that tensile strain might act on hPDLSCs differentiation, and the early response to mechanical stress should be investigated.

Material and methods: The hPDLSCs were cultured in vitro and isolated via a magnetic activated CD146 cell sorting system. After investigation of surface markers and other experiments for identification, hPDLSCs were subjected to cyclic tensile strain at 3,000 $\mu$ strain for $3 \mathrm{~h}, 6 \mathrm{~h}, 12 \mathrm{~h}$, and $24 \mathrm{~h}$, without addition of osteogenic supplements. In the control groups, the cells were cultured in similar conditions without mechanical stimulation. Then osteogenic related genes and proteins were analyzed by RT-PCR and western blot.

Results: Cyclic tensile strain at 3,000 $\mu$ strain of 6 h, 12 h, and 24 h durations significantly increased mRNA and protein expressions of Satb2, Runx2, and Osx, which were not affected in unloaded hPDLSCs.

Conclusions: We indicate that hPDLSCs might be sensitive to cyclic tensile strain. The significant increase of Runx2, Osx and Satb2 expressions may suggest an early response toward osteogenic orientation of hPDLSCs.

Key words: periodontal ligament stem cells, mechanical stress, osteogenesis.

\section{Introduction}

Human periodontal ligament stem cells (hPDLSCs) have been isolated and identified as mesenchymal adult stem cells (MSCs) since 2004 [1]. It has been shown that hPDLSCs are multipotent cells with features similar to bone marrow and dental pulp MSCs, which are capable of proliferating and producing different types of tissues such as bone, adipose and tooth associated-tissues in specific media [1, 2]. Periodontal ligament (PDL) has been found to be a ready and efficient autologous source of stem cells for tissue engineering in regenerative dentistry.

It has been well accepted that mechanical signals may regulate the direction of stem cell differentiation [3-5]. Tensile strain induces osteogenic diffe-
Corresponding author:

Linkun Zhang MD, PhD

Tianjin Stomatological Hospital

Nankai University

No. 75, Dagu Road

Tianjin 300041, China

Phone: +86-15900398050

E-mail: tangnaoh@sina.com 
rentiation of human bone-derived MSCs without addition of osteogenic supplements [6]. However, little is known about the response of PDL-derived MSCs to any type of mechanical stimulus. According to findings from previous experiments on MSCS derived from other sources, we hypothesized that tensile strain alone may induce osteogenic differentiation of hPDLSCs, and enhance osteogenic regulatory gene expression.

In the oral environment, PDL sensitively mediates the transmission of stress stimuli to the alveolar bone for periodontal tissue remolding [7]. Clinically, PDL distraction may successfully restore the adjacent alveolar bone [8-10]. Stretching-like stress has been found to increase expression of osteogenic genes and proteins such as alkaline phosphatase (ALP), bone morphogenetic protein (BMP)-2, BMP6, osterix (Osx), runt-related transcription factor-2 (RunX2), and MSX1 of human PDL cells [11-13]. Derived from PDL, hPDLSCs are surmised to have a similar osteogenic response induced by strain, in whose support little evidence has been noted as of this day.

Essential to cellular commitment to a differentiation lineage is the activation of defined transcription factors. Studies have revealed that Runx2 and Osx are two essential transcription factors in the osteogenic pathway [14-16]. Satb2, encoding a nu- clear matrix protein, is a novel transcriptional factor found expressed in cells of the osteoblast lineage [17]. Cell response toward osteogenic orientation could be suggested by the expression change of these related factors.

The objective of this study is to preliminarily examine the early response of hPDLSCs subject to a mechanical force. We investigated the effects of cyclic tensile strain of 3,000 ustrain on the osteogenic transcription factors in cultured hPDLSCs, and with different loading durations.

\section{Material and methods}

\section{Primary cell culture of human PDL cells}

Human PDL cells were obtained from 26 periodontally healthy, non-carious premolars extracted from 8 donors aged between 12 and 18 years for orthodontic reasons with informed consent. The periodontal ligament of each tooth was scratched off and soaked in sterilizing medium containing $100 \mathrm{U} / \mathrm{ml}$ penicillin and $100 \mathrm{mg} / \mathrm{ml}$ streptomycin, followed by digestion in $0.3 \%$ collagenase type I (Sigma, USA) with vigorous shaking for $40 \mathrm{~min}$ at $37^{\circ} \mathrm{C}$. Floating populations were removed by centrifugation at $1000 \mathrm{rpm}$ for 8 min with the cells pelleted. A single-cell suspension was obtained and re-suspended in alpha minimum essential medium ( $\alpha$-MEM; Gibco, USA) con-
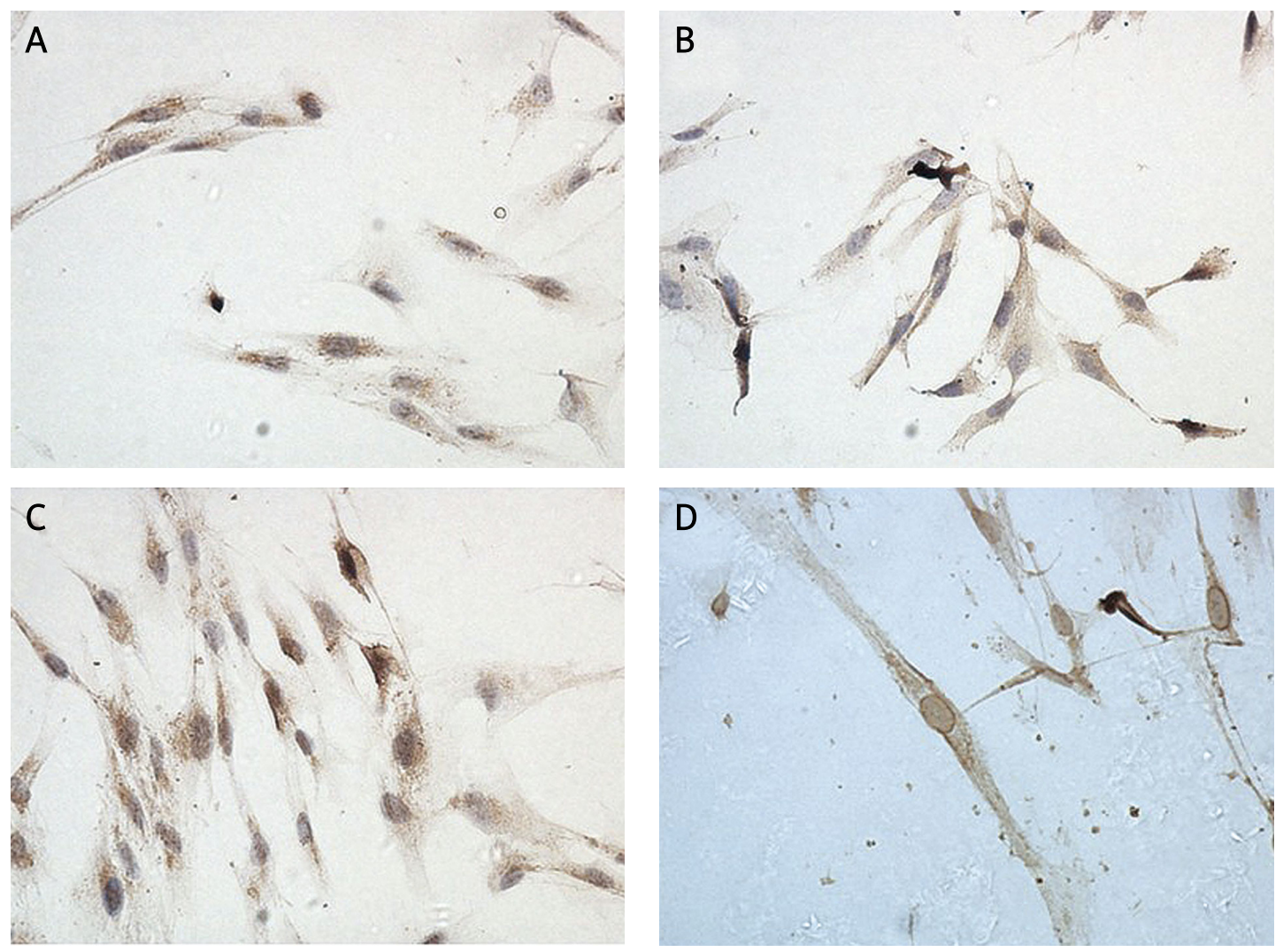

Figure 1. Immunohistochemical staining of surface makers on hPDLSCs. A - Stro-1. B - CD271. C - CD146. D - Scleraxis (Original magnification of all images 400x) 

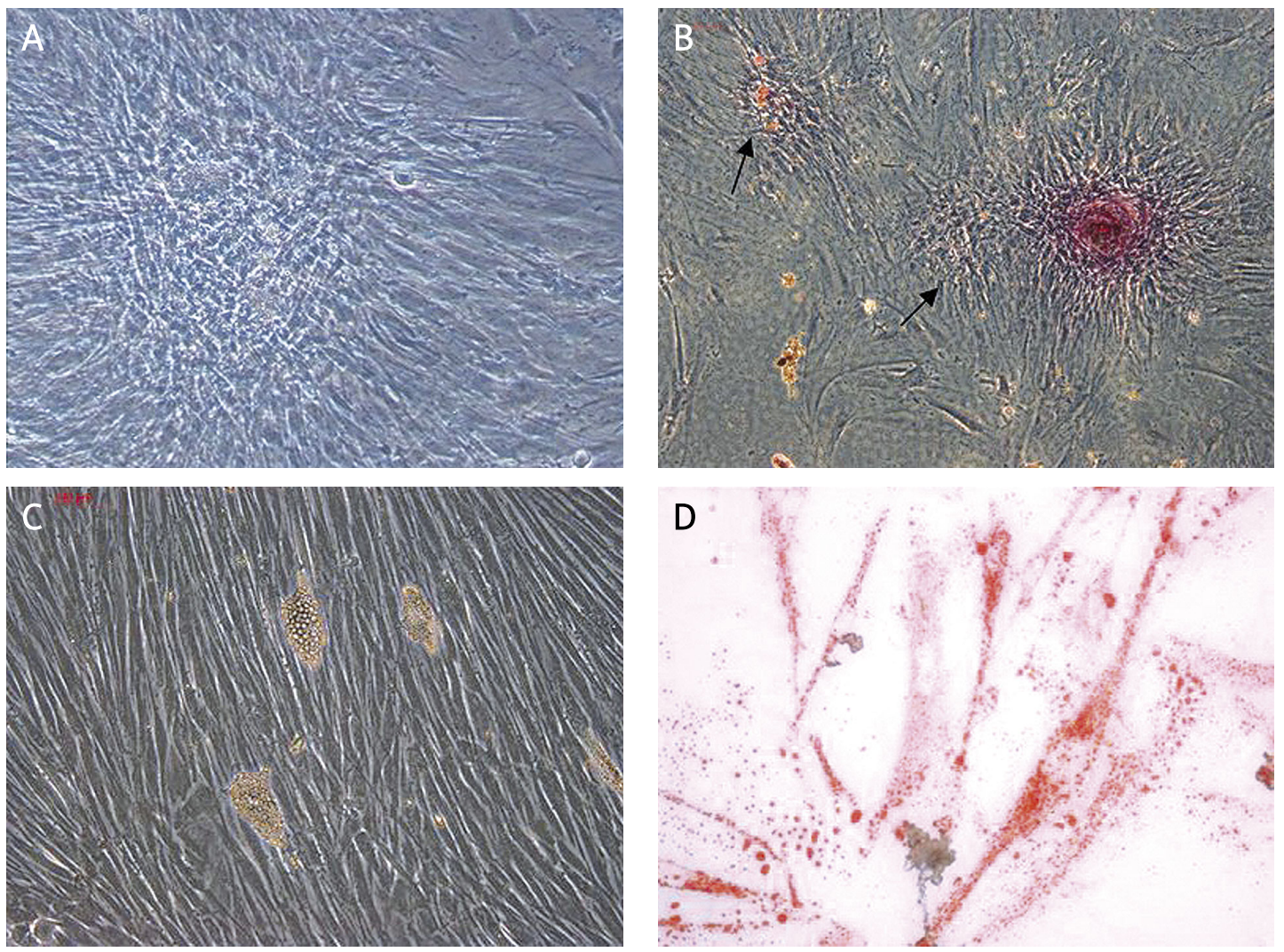

Figure 2. Osteogenic and adipogenic differentiation of $h$ PDLSCs. A, B -3 weeks after osteogenic induction. C, D - 2 weeks after adipogenic induction. B - Alizarin red staining and forming calcium nodules (arrows) (Original magnification of all images 200x). D - Oil red O staining and lipid drops (Original magnification of all images 400x)

taining 15\% (v/v) heat-inactivated fetal bovine serum (FBS; Hyclone, USA), $100 \mathrm{U} / \mathrm{ml}$ penicillin, and $100 \mathrm{mg} / \mathrm{ml}$ streptomycin. The cell suspension was seeded in new 25 - $\mathrm{ml}$ flasks and incubated at $37^{\circ} \mathrm{C}$ in humidified air with $5 \% \mathrm{CO}_{2}$. The medium was changed three times a week. On reaching confluence, the cells were trypsinized and serially passaged.

\section{Isolation and cultivation of hPDLSCs}

The hPDLSCs were isolated from the $3^{\text {rd }}$ passage $\mathrm{PDL}$ cells via a magnetic activated cell sorting system (Miltenyi Biotec, Germany). CD146 cell sorting columns (Miltenyi Biotec, Germany) were used to collect the CD146 (+) cells, the percentage of which in the total cell volume was about 1.5\%. CD146 (-) cells were collected as a control for cell identification. After centrifugation at $1000 \mathrm{rpm}$, the sorted cells were re-suspended in culture medium composed of $\alpha$-MEM (Gibco, USA) supplemented with 15\% (v/v) FBS (Hyclone, USA), and 0.3\% (v/v) bovine pituitary extract (BPE; M\&C Gene Technology, China), followed by cultivation in humidified air with $5 \% \mathrm{CO}_{2}$ at $37^{\circ} \mathrm{C}$. The medium was changed three times a week. On reaching confluence, the cells were passaged or seeded into dishes or plates for subsequent experiments. The CD146 (+) cells were identified as hPDLSCs (Figures 1-3).

\section{Application of cyclic tensile strain}

The hPDLSCs cultured through the $2^{\text {nd }}$ passage were used in our study. The hPDLSCs were detached and seeded into the force-loading plates at a density of $1 \times 10^{5} \mathrm{cells} / \mathrm{cm}^{2}$. The plates were made from the bottom part of $75 \mathrm{~cm}^{2}$ cell culture flasks (BD Falcon, USA), each measuring $3 \times 8 \mathrm{~cm}^{2}$ in area and $1.15 \mathrm{~mm}$ in thickness. After $48 \mathrm{~h}$ incubation and $24 \mathrm{~h}$ serum starvation, the cells on the plates were subjected to cyclic uniaxial tensile stress of 3,000 ustrain on a self-made four-point bending system $[18,19]$ at $0.5 \mathrm{~Hz}$ as reported before. The cells were loaded for 3 h, 6 h, 12 h, and 24 h, respectively. In the unloaded control groups, the cells were cultured on similar plates and kept in the same incubator simultaneously. For each group, we seeded hPDLSCs into three plates at one time and repeated experiments three times.

\section{RNA isolation and quantitative RT-PCR}

Samples were obtained from the independent experiments on loaded groups and controls. Samples of loaded groups were collected immediately after mechanical loading. The total RNA was extracted using Trizol (Invitrogen, USA) reagent according to the manufacturer's protocol. The concentration and purity of total RNA was confirmed by $260 / 280$ OD value of 1.8-2.0. 4.0 $\mu$ l of total RNA from each sample 

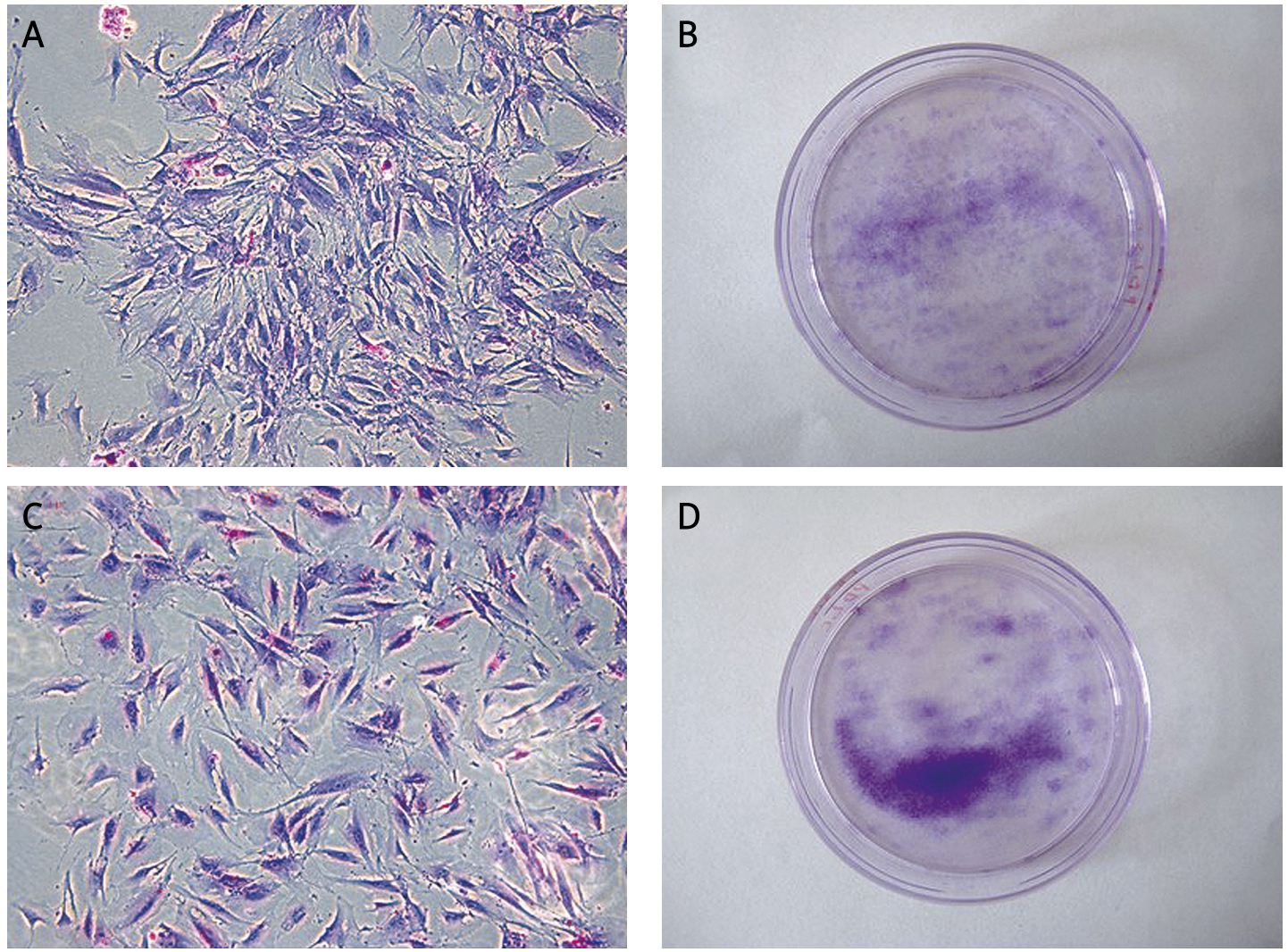

Figure 3. Cloning formation experiments of CD146+ PDL cells (A, B) and CD146- PDL cells (C, D)

were subject to reverse transcription using a SYBR PrimeScript RT-PCR Kit (Takara, Japan) according to the manufacturer's protocol [20]. Real-time PCR was performed for quantifying the mRNA levels in Satb2, Runx2, and Osx with a SYBR Premix Ex Taq ${ }^{\mathrm{TM}}$ II Kit (Takara, Japan) in an ABI PRISM 7300 Fast Real-Time System. The PCR primers used are listed in Table I. The housekeeping gene glyceraldehyde- 3 phosphate dehydrogenase (GAPDH) was used as an endogenous control. The starting copy number of each sample was calculated with 7300 System SDS software from the standard curve. The results of the target genes were normalized against GAPDH levels and the relative expressions calculated using the delta threshold cycle (CT) method. The cDNA of the unloaded control groups normalized against the GAPDH levels have been ascribed a fold induction of 1 . Melting curves for PCR were generated to ensure the purity of the amplification product.

\section{Western blot analysis}

To obtain whole-cell extracts, the cells were washed twice with ice-cold PBS and lysed in a lysis buffer (Keygen total protein extraction kit, Keygen Biotech, China). The cytosolic fraction was collected after centrifugation at $14000 \mathrm{rpm}$ at $4^{\circ} \mathrm{C}$ for $15 \mathrm{~min}$ and quantitatively assayed with the BCA method [21].

Table I. Real-time RT-PCR primers used in the experiments

\begin{tabular}{|llcc|}
\hline Target & Primer sequence & PCR product [bp] & GenBank Accession No. \\
\hline Satb2 & Forward: 5'-CCAGGAGTTTGGGAGATGGTAT-3' & 163 & NM_015265.2 \\
\cline { 2 - 4 } & Reverse: 5'-GTGAGGAGACTGTTCGTTGGTT-3' & & \\
\hline \multirow{2}{*}{ Runx2 } & Forward: 5'-CAGATGGGACTGTGGTTACTGT-3' & 169 & NM_ \\
\cline { 2 - 4 } & Reverse: 5'-GTGAAGACGGTTATGGTCAAGG-3' & & NM_152860.1 \\
\hline Osterix & Forward: 5'-ACCTACCCATCTGACTTTGCTC-3' & 125 & \\
\cline { 2 - 4 } & Reverse: 5'-CCACTATTTCCCACTGCCTTG-3' & & NM_002046.3 \\
\hline GAPDH & Forward: 5'-GGAAGGTGAAGGTCGGAGT-3' & 229 & \\
\cline { 2 - 4 } & Reverse: 5'-TGGAAGATGGTGATGGGATT-3' & & \\
\hline
\end{tabular}



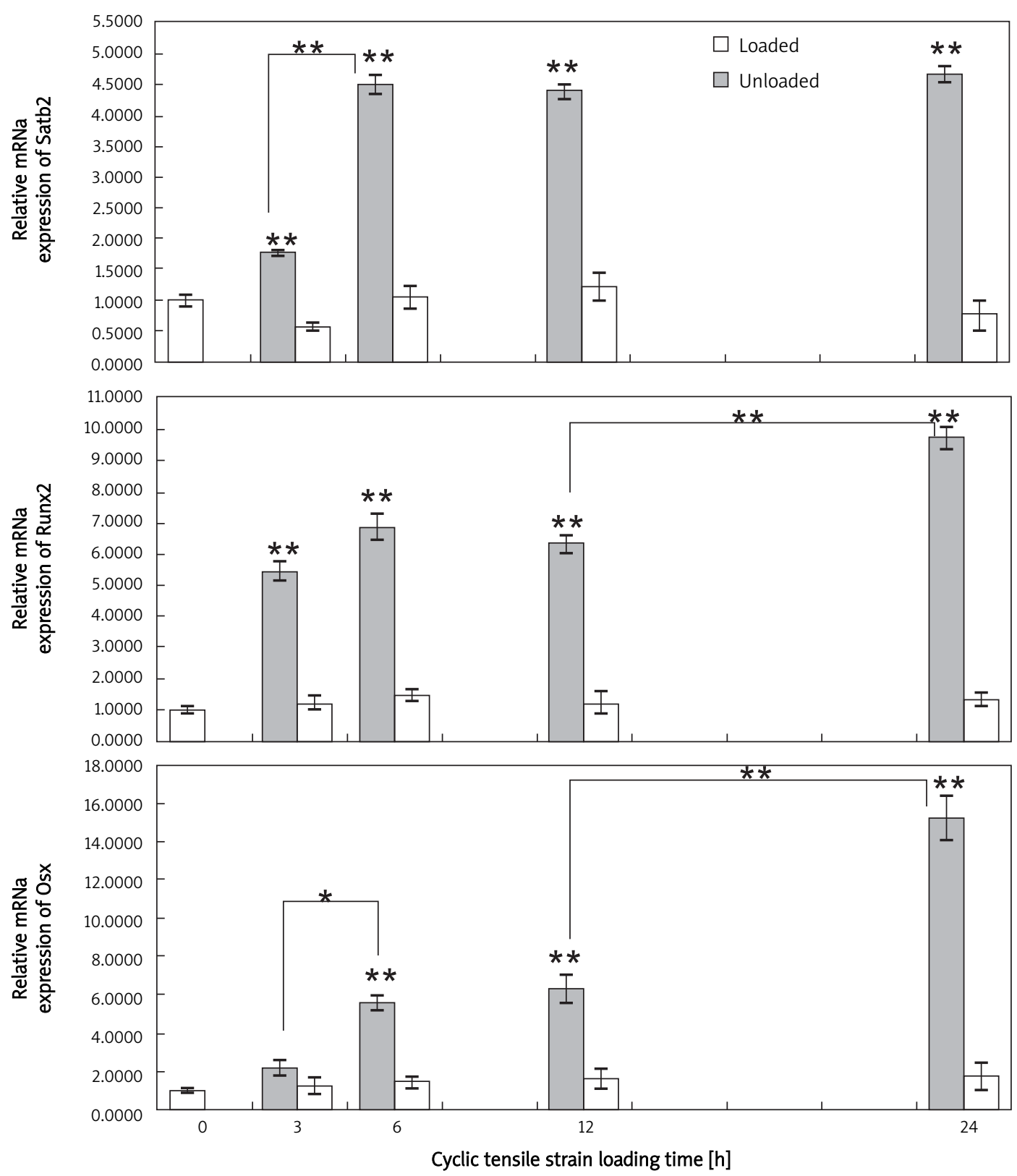

Figure 4. Effects of cyclic tensile strain on osteoblast-related genes (Satb2, Runx2, and Osx) of hPDLSCs. Bars represent mean \pm SD. Significant differences among the groups are noted by ${ }^{*} p<0.05,{ }^{* *} p<0.01$

An equal amount of protein for each sample was resolved using sodium dodecyl sulfate (SDS)-10\% polyacrylamide gel electrophoresis (PAGE) at $60 \mathrm{~V}$ for $5 \mathrm{~h}$, and then electrophoretically transferred onto a polyvinylidene difluoride (PVDF) membrane. The membrane was blocked with 5\% skim milk and sequentially incubated with $1: 1000$ dilutions of antiSATB2 (ab51502, Abcam, USA), anti-RUNX2 (ab23981, Abcam, USA), and anti-osterix (ab57335, Abcam, USA) antibodies, followed by addition of horseradish peroxidase (HRP)-conjugated secondary antibody (diluted 1 : 2000; Bioss, China). GAPDH was used as an internal control. Immunoreactive proteins were visualized using a chemiluminescence kit (Keygen Biotech, China). Band intensities were determined by average optical density (OD) analysis using the Image Pro-Plus software, and normalized against the GAPDH levels.

\section{Statistical analysis}

Statistical analysis was carried out with the SPSS 16.0 software (SPSS Inc., USA). Mean values and standard errors of the mean (SEM) were calculated. For multiple comparisons, one-way analysis of variance (ANOVA) followed by Scheffe post hoc test was used. The significance level was set at $p<0.05$. 

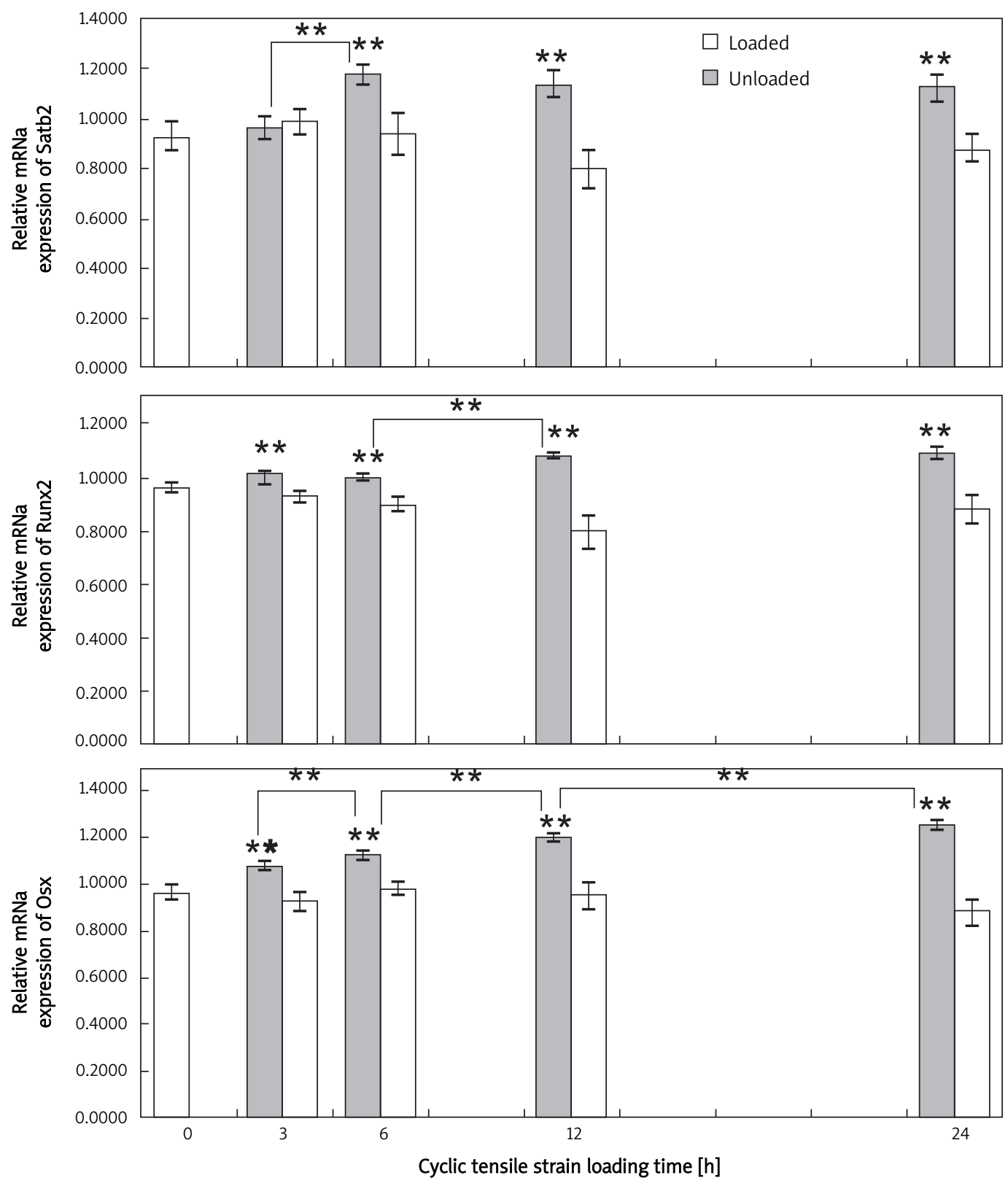

Figure 5. Effects of cyclic tensile strain on osteoblast-related proteins (SATB2, RUNX2 and osterix) of hPDLSCs. Bars represent mean \pm SD. Significant differences among the groups are noted by ${ }^{*} p<0.05,{ }^{* *} p<0.01$

\section{Results}

\section{Identification of hPDLSCS}

The CD146 $(+)$ cells were identified as hPDLSCs including Stro-1 (+), CD146 (+), CD271 (+), and Scleraxis $(+)$ by immunohistochemical staining (Figure 1 ). Furthermore, the cells cultured in the adipogenic or osteogenic medium differentiated into adipocytes and osteoblasts, respectively (Figure 2). The CD146 (+) cells had a clone-like growth characteristic with a cloning formation rate of $64.93 \%$ observed by crystal violet hydrate staining (Figure 3), whereas the CD146 (-) cells were Stro-1 (-), CD271 (-), and Scleraxis (-) with little cloning noted.

\section{mRNA expression of osteogenic transcription factors}

Significant differences $(p<0.01)$ between the loaded and unloaded cells were indicated in the mRNA expression levels of Satb2, Runx2, and Osx (Figure 4).

For Satb2 and Runx2, the mRNA levels increased significantly after a $3 \mathrm{~h}$ exposure to tensile strain. A significant increase in Satb2 expression after a $6 \mathrm{~h}$ exposure was marked when compared with that following a 3 h exposure, whereas no significant changes were observed between the groups loaded for $6 \mathrm{~h}, 12 \mathrm{~h}$ and $24 \mathrm{~h}$. Runx2 expression increased significantly after a $24 \mathrm{~h}$ exposure compared with that 
after a $12 \mathrm{~h}$ exposure, while the changes were insignificant between the groups loaded for $3 \mathrm{~h}, 6 \mathrm{~h}$ and $12 \mathrm{~h}$.

For Osx, the mRNA levels were significantly elevated after a $6 \mathrm{~h}$ exposure to tensile strain. The change was also significant between a loading duration of $24 \mathrm{~h}$ and one of $12 \mathrm{~h}$.

\section{Protein expression of osteogenic transcription factors}

Significant differences $(p<0.01)$ between cells loaded by cyclic tensile strain and unloaded were also indicated in the protein expression levels of SATB2, RUNX2, and osterix (Figure 5). The level of SATB2 increased significantly after a $3 \mathrm{~h}$ exposure to tensile strain, whereas the level of RUNX2 was significantly elevated following a $6 \mathrm{~h}$ exposure. The osterix expression saw stepwise increases when loaded for 3 h, 6 h, 12 h and 24 h. For osterix, significant differences $(p<0.01)$ were indicated between groups of varied loading durations.

\section{Discussion}

The PDL phorocytes consist of fibroblasts, osteoblasts, osteoclasts and undifferentiated mesenchymal cells. Human PDLSCs were first isolated and identified from human PDL by single-colony selection and magnetic activated cell sorting in 2004 [22].

In our study, hPDLSCs were successfully isolated by magnetic activated cell sorting with CD146 cell sorting columns. The results of phenotypic detection of PDLSCs were similar to previous studies [1, 2] showing that PDLSCs have similar phenotypes to bone marrow stem cells (BMSCs) with their pluripotency also proven. All the findings suggest that purified mesenchymal stem cells can be obtained from PDL, a readily available source for regenerative dentistry.

Laboratory evidence has already affirmed the significance of tensile strain on osteogenic differentiation of BMSCS. Jagodzinski et al. [23] found that cyclical stretching with elongation of $8 \%$ at $1 \mathrm{~Hz}$ for 3 days significantly up-regulated expression of ALP, osteocalcin, collagen type I and Runx2 of human BMSCs. Similarly, Friedl et al. [4] found that cyclic tensile strain at 3000 ustrain for 3 days significantly stimulated the expression levels of the osteogenic marker genes (Runx2, SPARC, SPP1, and ALPL). Other studies with 3D-cultured BMSCs [4, 24, 25] also verified cyclic tensile strain-induced osteogenic differentiation.

Our study aims to investigate the early response of hPDLSCs subject to cyclic tensile strain, which simulated a physiological stimulus. Determining the actual strain value in a microenvironment seems difficult [26]. In previous studies in vitro, cell deformation strain was used to evaluate stress loaded on cells. 10,000 $\mu$ strain equates to $1 \%$ strain of cells. Approximately, the deformation of PDL could be calculated based on the mobility of the crown and width of PDL, which means a wide range of $0 \%$ to
$25 \%$ in physical conditions. Yamaguchi [27] believed that strain from $9 \%$ to $24 \%$ simulated the states of PDL under an orthodontic force. However, cells in PDL bear much less strain than PDL tissue, thanks to the extracellular matrix, which is rich in elastic fibers, collagen and water, and may shield the cells from large deformation and loading [28]. Therefore, we used a cyclic uniaxial tensile strain of 3,000 $\mu$ strain, which was similar to what was employed in the Friedl et al. study [4], and lower than in some similar studies $[13,29,30]$, expecting to reach a certain supplementary understanding.

In order to investigate whether tensile strain could induce differentiation of hPDLSCs in an osteogenic orientation over the first $24 \mathrm{~h}$, we studied the expression levels of Runx2 and Osx. Unfortunately, identification of mRNA and protein markers characterizing osteogenic differentiation was complicated by the known variability of cells from different individuals, and varied harvesting methods and passages, as appears to be a common problem in cultured stem cell research. Therefore, in attaining identical characteristics in every group from the beginning, we employed the same passage of the same explants from age-specific adolescent donors.

Runx2 and Osx genes are two essential transcription factors for osteoblast differentiation and bone formation [31]. Null mutations of either leads to a complete absence of bone in mice [14, 16, 31, 32]. The way that Runx2 modulates osteogenic differentiation varies widely, and one possible mechanism is the interaction with the specific transcription factor Osx, which has been supposed to act downstream of Runx2 [33, 34]. Satb2 is another transcription factor modulating osteoblast differentiation acting as a multifunctional determinant $[17,35,36]$. Satb2 may promote bone formation by synergizing with Runx2 [17, 37] or repressing Homeobox a2 (Hoxa2), an inhibitor of bone formation [17]. Satb2 may also up-regulate expression of Runx2 by repressing Hoxa2 [35]. Our findings suggest that cyclic stretching alone at 3,000 ustrain could significantly up-regulate Runx2, Osx and Satb2 after $6 \mathrm{~h}$. This confirms the mechanosensitivity of hPDLSCs to tensile strain in the absence of chemical factors. Osteogenic differentiation of hPDLSCs might be started after loading for $3 \mathrm{~h}$ or $6 \mathrm{~h}$, and Satb2 might participate in this tension-induced differentiation.

Additionally, we noted that expression of Runx2 and Osx might present a time-dependent manner, whereas Satb2 maintained a relatively stable level after hPDLSCs were stretched for $6 \mathrm{~h}$. It is speculated that these three factors were affected by tensile strain in different ways, and the specific pathways involved call for further investigations.

One limitation to this study worth noting is that the loading plate is vulnerable to breakage after repeated deformation at 3,000 $\mu$ strain, which limits the loading time to no more than $30 \mathrm{~h}$ and ignores 
the response of cells to mechanical force after being loaded for more than 1 day, despite which, however, the results have failed to indicate that cyclic tensile strain for 6 h elevates expression of osteogenic factors of hPDLSCs significantly.

In conclusion, we find that hPDLSCs may be sensitive to cyclic tensile strain. Cyclic stretching at 3,000 ustrain of $6 \mathrm{~h}$ duration may significantly increase mRNA and protein expressions of Runx2, Osx and Satb2 in hPDLSCs, which suggests an early response toward osteogenic orientation. Based on the results of this and previous works, PDLSCs may be exploited as a readily available source for mechanical-forcefacilitated bone tissue engineering, which requires further investigations.

\section{Acknowledgments}

This work was supported by the Nature Science Foundation of China (No. 30470436 and No. 81030034) and the Youth Science Foundation of Sichuan (No. 08ZQ026-049).

\section{References}

1. Seo BM, Miura M, Gronthos S, et al. Investigation of multipotent postnatal stem cells from human periodontal ligament. Lancet 2004; 364: 149-55.

2. Trubiani O, Orsini G, Zini N, et al. Regenerative potential of human periodontal ligament derived stem cells on three-dimensional biomaterials: a morphological report. J Biomed Mater Res A 2008; 87: 986-93.

3. Pelaez D, Huang CY, Cheung HS. Cyclic compression maintains viability and induces chondrogenesis of human mesenchymal stem cells in fibrin gel scaffolds. Stem Cells Dev 2009 J 2009; 18: 93-102.

4. Friedl G, Schmidt H, Rehak I, Kostner G, Schauenstein K, Windhager R. Undifferentiated human mesenchymal stem cells (hMSCs) are highly sensitive to mechanical strain: transcriptionally controlled early osteo-chondrogenic response in vitro. Osteoarthritis Cartilage 2007; 15: 1293-300.

5. Thorpe SD, Buckley CT, Vinardell T, O'Brien FJ, Campbell VA, Kelly DJ. The response of bone marrow-derived mesenchymal stem cells to dynamic compression following TGFbeta3 induced chondrogenic differentiation. Ann Biomed Engl 2010; 38: 2896-909.

6. Sumanasinghe RD, Bernacki SH, Loboa EG. Osteogenic differentiation of human mesenchymal stem cells in collagen matrices: effect of uniaxial cyclic tensile strain on bone morphogenetic protein (BMP-2) mRNA expression. Tissue Eng 2006; 12: 3459-65.

7. Kawarizadeh A, Bourauel C, Götz W, Jäger A. Early responses of periodontal ligament cells to mechanical stimulus in vivo. J Dent Res 2005; 84: 902-6.

8. Kumar PS, Saxena R, Patil S, Keluskar KM, Nagaraj K, Kotrashetti SM. Clinical investigation of periodontal ligament distraction osteogenesis for rapid orthodontic canine retraction. Aust Orthod J 2009; 25: 147-52.

9. Lv T, Kang N, Wang C, Han X, Chen Y, Bai D. Biologic response of rapid tooth movement with periodontal ligament distraction. Am J Orthod Dentofacial Orthop 2009; 136: 401-11.

10. Wilmes B, Drescher D. Vertical periodontal ligament distraction - a new method for aligning ankylosed and displaced canines. J Orofac Orthop 2009; 70: 213-23.
11. Wongkhantee S, Yongchaitrakul T, Pavasant P. Mechanical stress induces osteopontin via ATP/P2Y1 in periodontal cells. J Dent Res 2008; 87: 564-8.

12. Cho JH, Lee SK, Lee JW, Kim EC. The role of heme oxygenase-1 in mechanical stress- and lipopolysaccharide-induced osteogenic differentiation in human periodontal ligament cells. Angle Orthod 2010; 80: 552-9.

13. Wescott DC, Pinkerton MN, Gaffey BJ, Beggs KT, Milne TJ, Meikle MC. Osteogenic gene expression by human periodontal ligament cells under cyclic tension. J Dent Res 2007; 86: 1212-6.

14. Nakashima K, Zhou X, Kunkel G, et al. The novel zinc finger-containing transcription factor osterix is required for osteoblast differentiation and bone formation. Cell 2002; 108: 17-29.

15. Liu J, Zhao Z, Li J, et al. Hydrostatic pressures promote initial osteodifferentiation with ERK1/2 not p38 MAPK signaling involved. J Cell Biochem 2009; 107: 224-32.

16. Otto F, Thornell AP, Crompton T, et al. Cbfa1, a candidate gene for cleidocranial dysplasia syndrome, is essential for osteoblast differentiation and bone development. Cell 1997; 89: 765-71.

17. Dobreva G, Chahrour M, Dautzenberg M, et al. SATB2 is a multifunctional determinant of craniofacial patterning and osteoblast differentiation. Cell 2006; 125: 971-86.

18. Wang Y, Zhao Z, Li Y, et al. Up-regulated alpha-actin expression is associated with cell adhesion ability in 3-D cultured myocytes subjected to mechanical stimulation. Mol Cell Biochem 2010; 338: 175-81.

19. Li Y, Zhao Z, Song J, et al. Cyclic force upregulates mechano-growth factor and elevates cell proliferation in 3D cultured skeletal myoblasts. Arch Biochem Biophys 2009; 490: 171-6.

20. Yang $X$, Gong P, Lin Y, et al. Cyclic tensile stretch modulates osteogenic differentiation of adipose-derived stem cells via the BMP-2 pathway. Arch Med Sci 2010; 6: 152-9.

21. Li J, Zhao Z, Yang J, et al. p38 MAPK mediated in compressive stress-induced chondrogenesis of rat bone marrow MSCs in 3D alginate scaffolds. J Cell Physiol 2009; 221: 609-17.

22. Seo BM, Miura M, Gronthos S, et al. Investigation of multipotent postnatal stem cells from human periodontal ligament. Lancet 2004; 364: 149-55.

23. Jagodzinski M, Drescher M, Zeichen J, et al. Effects of cyclic longitudinal mechanical strain and dexamethasone on osteogenic differentiation of human bone marrow stromal cells. Eur Cells Mater 2004; 16: 35-41.

24. Kearney EM, Farrell E, Prendergast PJ, Campbell VA. Tensile strain as a regulator of mesenchymal stem cell osteogenesis. Ann Biomed Eng 2010; 38: 1767-79.

25. Sumanasinghe RD, Osborne JA, Loboa EG. Mesenchymal stem cell-seeded collagen matrices for bone repair: effects of cyclic tensile strain, cell density, and media conditions on matrix contraction in vitro. J Biomed Mater Res A 2009; 88: $778-86$.

26. Ren Y, Maltha JC, Kuijpers-Jagtman AM. Optimum force magnitude for orthodontic tooth movement: a systematic literature review. Angle Orthod 2003; 73: 86-92.

27. Yamaguchi M, Shimizu N. Identification of factors mediating the decrease of alkaline phosphatase activity caused by tension-force in periodontal ligament cells. Gen Pharmacol 1994; 25: 1229-35.

28. Jónsdóttir SH, Giesen EB, Maltha JC. Biomechanical behaviour of the periodontal ligament of the beagle dog during the first 5 hours of orthodontic force application Eur J Orthod 2006; 28: 547-52.

29. Pinkerton MN, Wescott DC, Gaffey BJ, Beggs KT, Milne TJ, Meikle MC. Cultured human periodontal ligament cells constitutively express multiple osteotropic cytokines and 
growth factors, several of which are responsive to mechanical deformation. J Periodontal Res 2008; 43: 343-51.

30. Myokai F, Oyama M, Nishimura F, et al. Unique genes induced by mechanical stress in periodontal ligament cells. J Periodontal Res 2003; 38: 255-61.

31. Eriksen EF. Cellular mechanisms of bone remodeling. Rev Endocr Metab Disord 2010; 11: 219-27.

32. Nakashima K, de Crombrugghe B. Transcriptional mechanisms in osteoblast differentiation and bone formation. Trends Genet 2003; 19: 458-66.

33. Nishio Y, Dong Y, Paris M, O'Keefe RJ, Schwarz EM, Drissi $\mathrm{H}$. Runx2-mediated regulation of the zinc finger Osterix/Sp7 gene. Gene 2006; 372: 62-70.

34. Sun DM, Liu ZB, Zhao Y, et al. Runx2 is involved in regulating osterix promoter activity and gene expression. Prog Biochem Biophys 2006; 33: 957-64.

35. Huang W, Yang S, Shao J, Li YP. Signaling and transcriptional regulation in osteoblast commitment and differentiation. Front Biosci 2007; 12: 3068-92.

36. Brewer E, Zhang J, Tu O, Tang J, Chen J. SATB2 overexpression promotes osteoblast differentiation and enhances regeneration of bone defects. J Bone Miner Res 2008; 23: S256.

37. Hassan MQ, Gordon JAR, Beloti MM, et al. A network connecting Runx2, SATB2, and the miR-23a 27a 24-2 cluster regulates the osteoblast differentiation program. Proc Natl Acad Sci U S A 2010; 107: 19879-84. 